\title{
4 A Generalization of a Sigma Coordinate Ocean Model and an Intercomparison of Model Vertical Grids
}

\author{
George Mellor $^{1}$, Sirpa HäKKinen ${ }^{2}$, Tal Ezer ${ }^{1}$ And Richard Patchen ${ }^{3}$ \\ ${ }^{1}$ Atmospheric and Oceanic Sciences Program Princeton University, Princeton, NJ \\ ${ }^{2}$ NASA Goddard Space Flight Center, Greenbelt, MD \\ ${ }^{3}$ Dynalysis of Princeton, Princeton N.J.
}

\subsection{Introduction}

Numerical ocean models increasingly make use of $\sigma$ - coordinate systems. A paper by Gerdes (1993) shows that these coordinate systems can be more general; he termed the generalized form an "s - coordinate" system. The main advantage of the $\sigma$ or s - system is that, when cast in a finite difference form, a smooth representation of the bottom topography is obtained; one can also easily incorporate a bottom boundary layer as well as a surface boundary layer in those coordinate systems. This is intuitively appealing and Gerdes has shown that superior numerical results are obtained relative to a $\mathrm{z}$ - level system. However, in regions of steep topography and crude resolution - a limiting case would be a seamount represented by a single grid point surrounded by a flat bottom - the so-called sigma coordinate pressure gradient error exists (Haney 1991, Mellor et al. 1994, 1998) and at least locally a $\mathrm{z}$ - level coordinate system might be preferred. On the other hand, in a recent study, Bell (1997) has shown that the step structure of $z$ - level models lead to vorticity errors and consequent errors in the barotropic component of the flow which, he reports, cause rather large temperature errors $\left(3\right.$ to $\left.4^{\circ} \mathrm{C}\right)$ on a $1^{\circ} \mathrm{x} 1^{\circ}$ grid of an Atlantic Ocean model after 3 months of integration. And it is difficult to model bottom boundary layers in a $\mathrm{z}$ - level model (Winton et al. 1998).

The pioneer Bryan-Cox model (Bryan 1969, Cox 1984) is a $\mathrm{z}$ - level model. A modification by Spall and Robinson (1993) is termed a "hybrid" coordinate system; they describe it as a $\mathrm{z}$ - level system in the region, $0>\mathrm{z}>-\mathrm{z}_{\mathrm{c}}=$ constant, and a $\sigma$ system when $\mathrm{z}_{\mathrm{c}}>\mathrm{z}>-\mathrm{H}(\mathrm{x}, \mathrm{y})$ where the transformed sigma equations apply. Presumably, this system is adopted so that surface mixed layers, which do not scale on depth, may be best represented. However, the hybrid system would appear to require separate numerical implementations for the two regions; their objectives can be realized more simply with the s - coordinate system described here.

For the sigma coordinate system of the Princeton Ocean Model (henceforth $\mathrm{POM}$ ), the top numerical level, $\mathrm{k}=1$, follows the free sea surface and the lowest numerical level, $\mathrm{k}=\mathrm{kb}$, follows the bottom depth; for $1<\mathrm{k}<\mathrm{kb}$, the distance 
between levels are in fixed proportion to each other independent of elevation or depth. In this paper we revise POM so that it is basically an s - coordinate system (although the variable, s, is, for very good reason, different from that used by Gerdes) and the proportionality constraint is removed. However, unlike the Gerdes formulation, the derivation and implementation permit a free surface and the distance between levels can change in time. Also the structure of the numerical algorithm is designed to support a wide variety of vertical coordinate systems including a $z$ level system. The present model generalization permits a $z$ - level representation everywhere in the model domain or locally in selected regions of the domain.

Song and Haidvogel (1994) have adopted many of the characteristics of POM but have also generalized to an s - coordinate system. Our formulation and implementation strategy, as described below, differs from theirs and we include the $z$ level option. Intercomparisons between different models have been the focus of recent projects (DYNAMO 1997, Willems et al. 1994).

The intercomparisons of this paper isolate the effect of different vertical grids; otherwise model physics and numerics are identical.

\subsection{The Governing Equations}

We first restrict attention to the analytic description of the basic equations although it is our intention that they be cast in finite difference form and we condition the description with that in mind. The basic equations described in an $(x, y, z)$ Cartesian coordinate system for the velocity components, U, V and W, and for potential temperature and salinity, $\mathrm{T}$ and $\mathrm{S}$, are

$$
\begin{gathered}
\mathfrak{I}(1)=0 \\
\mathfrak{I}(U)-f V+g \frac{\partial \eta}{\partial x}+\frac{g}{\rho_{o}} \int_{z}^{\eta}\left[\frac{\partial \rho^{\prime}}{\partial x}\right] d z^{\prime}=\frac{\partial}{\partial z}\left[K_{M} \frac{\partial U}{\partial z}\right]+F_{x} \\
\mathfrak{I}(V)+f U+g \frac{\partial \eta}{\partial y}+\frac{g}{\rho_{o}} \int_{z}^{\eta}\left[\frac{\partial \rho^{\prime}}{\partial y}\right] d z^{\prime}=\frac{\partial}{\partial z}\left[K_{M} \frac{\partial V}{\partial z}\right]+F_{y} \\
\mathfrak{I}(T)=\frac{\partial}{\partial z}\left[K_{H} \frac{\partial T}{\partial z}\right]+F_{T}-\frac{\partial R}{\partial z} \\
\mathfrak{I}(S)=\frac{\partial}{\partial z}\left[K_{H} \frac{\partial S}{\partial z}\right]+F_{S}
\end{gathered}
$$

and for twice the turbulence kinetic energy, $\mathrm{q}^{2}$ and length scale, $l$, we have 


$$
\begin{gathered}
\mathfrak{I}\left(q^{2}\right)=\frac{\partial}{\partial z}\left[K_{q} \frac{\partial q^{2}}{\partial z}\right] \\
+2 K_{M}\left[\left(\frac{\partial U}{\partial z}\right)^{2}+\left(\frac{\partial V}{\partial z}\right)^{2}\right]+\frac{2 g}{\rho_{o}} K_{H} \frac{\partial \tilde{\rho}}{\partial z}-\frac{2 q^{3}}{B_{1} l}+F_{q} \\
\mathfrak{I}\left(q^{2} l\right)=\frac{\partial}{\partial z}\left[K_{q} \frac{\partial q^{2} l}{\partial z}\right] \\
+E_{1} l\left(\mathrm{~K}_{M}\left[\left(\frac{\partial U}{\partial z}\right)^{2}+\left(\frac{\partial V}{\partial z}\right)^{2}\right]+E_{3} \frac{g}{\rho_{o}} K_{H} \frac{\partial \rho^{2}}{\partial z}\right)-\frac{q^{3}}{B_{1}} \tilde{W}+F_{l} \\
\mathfrak{I}(\varphi) \equiv \frac{\partial \varphi}{\partial t}+\frac{\partial U \varphi}{\partial x}+\frac{\partial V \varphi}{\partial y}+\frac{\partial W \varphi}{\partial z}
\end{gathered}
$$

In the above, $x$ and $y$ are horizontal coordinates (to be cast in a curvilinear, orthogonal coordinate system in section 4.4.) and $z$ is the vertical coordinate; $g$ is the gravity constant and $f$ is the Coriolis parameter. The density is $\rho$ whereas $\partial \tilde{\rho} / \partial z$ is the vertical density gradient corrected for adiabatic lapse rate. $\partial R / \partial z$ on the right hand side of (4) is the divergence of radiation flux, $R$. The vertical mixing coefficients are $K_{M}, K_{H}$, and $K_{q}$ and are functions of a Richardson number dependent on density stratification (Mellor and Yamada, 1982, Galperin et al, 1988 and Mellor, 1996). $\tilde{W}$ in (7) is a "wall proximity function". Horizontal diffusion fluxes are represented by $F_{\alpha}(\alpha=x, y, T, S, q, l)$.

\subsection{Transformation to the $s$-coordinate system}

The Cartesian coordinate system, $(x, y, z, t)$, is transformed to the $s$ - coordinate system, $\left(x^{*}, y^{*}, k, t^{*}\right)$, according to

$$
\begin{gathered}
x=x^{*} \\
y=y^{*} \\
t=t^{*} \\
z=\eta\left(x^{*}, y^{*}, t^{*}\right)+s\left(x^{*}, y^{*}, k, t^{*}\right)
\end{gathered}
$$

where $k$ is a continuous variable in the range, $1 \leq k \leq k b$, but when the differential equations are discretized, $k$ will also be discrete and will be the label of the numerical level. This usage is quite convenient and obviates the need for an intermediate variable [which Gerdes called $s$, and is not the usage in (9d)]. We define $\eta$ 
to be the surface elevation so that we will require $s=0$ at $k=1$. The key attribute of the transformation is $(9 \mathrm{a}, \mathrm{b})$; i.e., the horizontal coordinates in the Cartesian coordinate system are equated to the horizontal coordinates in the sigma system. Otherwise, the transformation is fairly general and includes, for example, a $z$ - level system, $s=\sigma(k)\left(H_{\text {max }}+\eta(x, y, t)\right)$, and the sigma system, $s=\sigma(k)(H(x, y)+$ $\eta(x, y, t)) ; H(x, y)$ is the bathymetry and $H_{\max }$ is its maximum value. In both cases, $\sigma(1)=0$ and $\sigma(\mathrm{kb})=-1$. In the case of the $z$ - level system (really a quasi $z$ - level system because $s$ is a function of time, but it is a weak function of time since, generally, $\eta / H<<1$ ), levels near the bottom are masked to approximate the bathymetry and, thus, $k b$ is a function of the horizontal coordinates. The distinguishing feature of both of these limiting cases is that $k$ is functionally separated from $x, y$ and $t$. In general they need not be separable as we shall demonstrate.

There are two ways of deriving the $s$ - coordinate equations. The first way is to apply integral (control volume) equations to a volume element bounded by $x^{*}, y^{*}$, $k$ and $x^{*}+\Delta x^{*}, y^{*}+\Delta y^{*}, k+\Delta k$. The second way is to formally transform equations (1)-(5). Thus, if $\phi$ is any dependent variable such that $\phi(x, y, z, t)=\phi^{*}\left(x^{*}, y^{*}\right.$, $\left.k, t^{*}\right)$ then

$$
\begin{gathered}
\frac{\partial \phi}{\partial x}=\frac{\partial \phi^{*}}{\partial x^{*}}+\frac{\partial \phi^{*}}{\partial k} \frac{\partial k}{\partial x}, \quad \frac{\partial \phi}{\partial y}=\frac{\partial \phi^{*}}{\partial y^{*}}+\frac{\partial \phi^{*}}{\partial k} \frac{\partial k}{\partial y} \\
\frac{\partial \phi}{\partial z}=\frac{\partial \phi^{*}}{\partial k} \frac{\partial k}{\partial z}, \quad \frac{\partial \phi}{\partial t}=\frac{\partial \phi}{\partial t}+\frac{\partial \phi^{*}}{\partial k} \frac{\partial k}{\partial t}
\end{gathered}
$$

From the fact that $\partial z / \partial x=0$, we obtain $\partial k / \partial x=-\left(\eta_{x}+s_{x}\right) / s_{k}$ where $s_{x} \equiv \partial s / \partial x^{*} \quad$ and $\quad \eta_{x} \equiv \partial \eta / \partial x^{*}$. Similarly, $\partial k / \partial y=-\left(\eta_{y}+s_{y}\right) / s_{k}$, $\partial k / \partial z=-1 / s_{k}$ and $\partial k / \partial t=-\left(\eta_{t}+s_{t}\right) / s_{k}$. (In discretized form, $s_{k} \equiv \delta s / \delta k$ is simply the distance, $\delta s$, between levels since $\delta k=1$. ) Therefore,

$$
\begin{gathered}
\frac{\partial \phi}{\partial x}=\frac{\partial \phi^{*}}{\partial x^{*}}-\frac{\partial \phi^{*}}{\partial k} \frac{s_{x}+\eta_{x}}{s_{k}}, \quad \frac{\partial \phi}{\partial y}=\frac{\partial \phi^{*}}{\partial y^{*}}-\frac{\partial \phi^{*}}{\partial k} \frac{s_{y}+\eta_{y}}{s_{k}} \\
\frac{\partial \phi}{\partial z}=\frac{\partial \phi^{*}}{\partial k} \frac{1}{s_{k}}, \quad \frac{\partial \phi}{\partial t}=\frac{\partial \phi^{*}}{\partial t^{*}}-\frac{\partial \phi^{*}}{\partial k} \frac{s_{t}+\eta_{t}}{s_{k}}
\end{gathered}
$$

We define a new variable, $\omega$, such that

$$
\omega \equiv W-\left(s_{x}+\eta_{x}\right) U-\left(s_{y}+\eta_{y}\right) V-\left(s_{t}+\eta_{t}\right)
$$


Note that the surface kinematic condition, $W=\eta_{x} U+\eta_{y} V+\eta_{t}$, is satisfied if we require that $\omega=0$ where $s=0$. Similarly, at the bottom, $W=-H_{x} U-H_{y} V$, and we set $\omega=0$ where $s=-(H+\eta)$.

If we use (11a, b, c, d) and (12) in equations (1) through (9) and drop all of the asterisks from independent and dependent variables, we obtain,

$$
\begin{gathered}
\mathfrak{I}(1)=0 \\
\mathfrak{I}(U)-f V s_{k}+g s_{k} \frac{\partial \eta}{\partial x}+g \frac{s_{k}}{\rho_{o}} \int_{k}^{0}\left[s_{k} \frac{\partial \rho^{\prime}}{\partial x}-\left(s_{x}+\eta_{x}\right) \frac{\partial \rho^{\prime}}{\partial k^{\prime}}\right] d k^{\prime} \\
=\frac{\partial}{\partial k}\left[\frac{K_{M}}{s_{k}} \frac{\partial U}{\partial k}\right]+\left(\frac{\partial s_{k} t_{x x}}{\partial x}+\frac{\partial s_{k} t_{x y}}{\partial y}\right) \\
\mathfrak{I}(V)+f U s_{k}+g s_{k} \frac{\partial \eta}{\partial y}+g \frac{s_{k}}{\rho_{o}} \int_{k}^{0}\left[s_{k} \frac{\partial \rho^{\prime}}{\partial y}-\left(s_{y}+\eta_{y}\right) \frac{\partial \rho^{\prime}}{\partial k^{\prime}}\right] d k^{\prime} \\
=\frac{\partial}{\partial k}\left[\frac{K_{M}}{s_{k}} \frac{\partial V}{\partial k}\right]+\left(\frac{\partial s_{k} t_{x y}}{\partial x}+\frac{\partial s_{k} t_{y y}}{\partial y}\right) \\
\mathfrak{I}(T)=\frac{\partial}{\partial k}\left[\frac{K_{H}}{s_{k}} \frac{\partial T}{\partial k}\right]+\left(\frac{\partial s_{k} q_{T}}{\partial x}+\frac{\partial s_{k} q_{T}}{\partial y}\right)-\frac{\partial R}{\partial k} \\
\mathfrak{I}\left(q^{2} l\right)=\frac{\partial}{\partial k}\left[\frac{K_{q}}{s_{k}} \frac{\partial q^{2} l}{\partial k}\right] \\
\mathfrak{I}(S)=\frac{\partial}{\partial k}\left[\frac{K_{H}}{s_{k}} \frac{\partial S}{\partial k}\right]+\left(\frac{\partial s_{k} q_{S}}{\partial x}+\frac{\partial s_{k} q_{S}}{\partial y}\right) \\
=\frac{2 \mathrm{~K}_{M}}{s_{k}}\left[\left(\frac{\partial U}{\partial k}\right)^{2}+\left(\frac{\partial V}{\partial k}\right)^{2}\right]+\frac{2 g}{\rho_{o}} K_{H} \frac{\partial \tilde{\rho}}{\partial k}-\frac{2 s_{k} q^{3}}{B_{1} l}+F_{q} \\
\left.\frac{s_{q}}{s_{k}} \frac{\partial q^{2}}{\partial k}\right] \\
=
\end{gathered}
$$

where now we define

$$
\mathfrak{I}(\varphi) \equiv \frac{\partial s_{k} \varphi}{\partial t}+\frac{\partial U s_{k} \varphi}{\partial x}+\frac{\partial V s_{k} \varphi}{\partial y}+\frac{\partial W \varphi}{\partial k}
$$




$$
\begin{gathered}
\text { In the above, } \quad t_{x x}=A_{M}(2 \partial U / \partial x), \quad t_{y y}=A_{M}(2 \partial V / \partial y), \\
t_{x y}=A_{M}(\partial U / \partial y+\partial V / \partial x), \quad q_{T x}=A_{H}(\partial T) / \partial x, \quad q_{T y}=A_{H} \partial T / \partial y \text { and } \\
q_{S x}=A_{H} \partial S / \partial x, \quad q_{S y}=A_{H} \partial S / \partial y . \text { Horizontal diffusion for } q^{2} \text { and } q^{2} l \text { is }
\end{gathered}
$$
the same form as that for $\mathrm{T}$ and $\mathrm{S}$. We now note that the gradients on the right sides of the momentum and scalar fluxes are reckoned along $k$ surfaces. However, any (simple) alternative, say, to transform so that gradients are taken along horizontal or isopycnal surfaces, leads to problems in properly modeling the bottom boundary layer (Mellor and Blumberg 1985) and possibly the surface layer. In the case of isopycnal surfaces large vertical flux terms normal to top or bottom surfaces can be created and can compete with the vertical mixing parameterization denoted by $K_{M}$ and $K_{H}$; these recognize that turbulent motion normal to surfaces are small whereas $A_{M}$ and $A_{H}$ are not. On the other hand, mixing along $k$ surfaces creates problems in, say, the interior ocean in the presence of vertical temperature or salinity gradients; this will be discussed below.

Values for the horizontal viscosity, $A_{M}$, and diffusivity, $A_{H}$, will play an important role in the solutions to be discussed below.

\subsection{Finite Difference Equations}

The differential equations are first written in finite volume form, that is, the equations are integrated over a finite cell volume. In particular for the advective operator,

$$
\iiint \mathfrak{I}(\varphi) \delta V=\frac{\partial}{\partial t} \iiint \varphi \delta V+\iint \varphi U \cdot \mathbf{n} \delta A
$$

where $\delta V$ is an elemental volume, $\delta A$ an elemental area and $\mathbf{n}$ a unit vector normal to the area. Then

$$
\begin{gathered}
\mathfrak{I}(1)=0 \\
\mathfrak{I}(U)-f A V \delta s+g \delta y \delta s \delta_{x} \eta+g \delta y \delta s \sum_{k}\left[\delta s \delta_{x} \rho-\left(\delta_{x} s+\delta_{x} \eta\right) \delta_{k} \rho\right] \\
=A \delta_{k}\left[K_{M} \frac{\delta_{k} U}{\delta s}\right]+\delta_{x}\left(t_{x x} \delta s \delta y\right)+\delta_{y}\left(t_{x y} \delta s \delta x\right) \\
\mathfrak{I}(V)+f A U \delta s+g \delta x \delta s \delta_{y} \eta+g \delta x \delta s \sum_{k}\left[\delta s \delta_{y} \rho-\left(\delta_{y} s+\delta_{y} \eta\right) \delta_{k} \rho\right] \\
=A \delta_{k}\left[K_{M} \frac{\delta_{k} V}{\delta s}\right]+\delta_{x}\left(t_{x y} \delta s \delta y\right)+\delta_{y}\left(t_{y y} \delta s \delta x\right)
\end{gathered}
$$




$$
\begin{aligned}
& \mathfrak{I}(T)=A \delta_{k}\left[K_{H} \frac{\delta_{k} T}{\delta s}\right]+\delta_{x}\left(q_{T x} \delta s \delta y\right)+\delta_{y}\left(q_{T y} \delta s \delta x\right) \\
& \mathfrak{I}(S)=A \delta_{k}\left[K_{H} \frac{\delta_{k} S}{\delta s}\right]+\delta_{x}\left(q_{S x} \delta s \delta y\right)+\delta_{y}\left(q_{S y} \delta s \delta x\right)
\end{aligned}
$$

where now we define

$$
\mathfrak{I}(\varphi) \equiv \frac{A \delta_{t}(\delta s \varphi)}{\delta t}+\delta_{x}(\delta s \delta y U \varphi)+\delta_{y}(\delta s \delta x V \varphi)+A \delta_{k}(\omega \varphi)
$$

This formulation accommodates an orthogonal, curvilinear, horizontal coordinate system; the elements of each cell have the horizontal dimensions, $\delta x, \delta y$ and the area, $A \equiv \delta x \delta y$. The vertical dimension is $\delta s$. Formally, there are additional horizontal viscous terms in (22) and (23) due to curvature, but these have been neglected since the terms themselves are highly empirical and we prefer the values, $A_{M}$ and $A_{H}$, be as small as possible. In the calculations discussed below, the horizontal coordinates will be spherical, a special case of more general orthogonal curvilinear coordinates accommodated by the model.

\subsection{Model Strategy}

The algorithmic structure of the generalized model does not differ greatly from the previous POM (Blumberg and Mellor 1987, Mellor 1996) in that it has a free surface, split internal, external time steps and second order horizontal differencing on a staggered $\mathrm{C}$ grid (although work on other differencing schemes is in progress) and an imbedded turbulence closure scheme which primarily governs surface and bottom boundary layers. The new features are that the more general vertical increment, $\delta s(i, j, k, n)$, where $i, j, k$ are the spatial indices and $n$ is the time index, has replaced the sigma increment, $\delta s(k)(H(i, j)+\eta(i, j, n))$ and the previous two-dimensional land mask is now three-dimensional to accommodate the $z$ - level grid when that system is invoked. All of the code except for one subroutine is universal. The exceptional subroutine is crafted to produce whichever coordinate system is desired. In the examples discussed below, there are three such subroutines (SUBROUTINE MAKSIG, for example), one for each of the three grids we have chosen to test in this paper. The active subroutine is called at each internal mode time step. 


\subsection{Numerical Simulations Comparing Three Vertical Coordinate Systems}

We will examine calculations using three vertical grids, a $z$ - level grid, a $\sigma$ grid and a generalized $\sigma$ grid. Fig. 4.1 illustrates the domain used in the calculations which includes a bowl shaped deep basin in the north, east and west and a vertical wall at the equator; the basin connects to a shallow continental shelf in the north. Fig. 4.1 a represents both the sigma and generalized sigma grids. The horizontal resolution of all the experiments is about $1^{\circ} \times 1^{\circ}$ on a $51 \times 76$ horizontal grid. There are 21 vertical grid levels with spacing decreasing near the surface; of course, for the $z$ - level grid, levels are truncated for all but the deepest depths (Fig. 4.1 b). All vertical walls including those of the $z$ - level steps are adiabatic and frictionless.

The initial temperature is $T(x, y, z)=T(z)=5+15 \exp (z / 1000 m)$ and the applied zonal wind stress field is $\left(\tau_{x o}, \tau_{y o}\right)=\left(-.0002 \mathrm{~m}^{2} \mathrm{~s}^{-2} \sin \{2 \pi(\phi / 60)\}, 0\right)$ where $f$ is latitude. The surface heat and salinity flux and penetrative radiation are nil.

Fig. 4.2 shows the three vertical grids in a north-south cross section at the center of the domain shown in Fig. 4.1 . For the z-level grid (Fig. 4.2 a), we have a conventional grid with the characteristic step structure at the bottom. For the conventional sigma grid (Fig. 4.2 b), the spacing between the different layers have the same proportion independent of depth, and the lowest layer smoothly follows the bottom topography. For the generalized sigma grid (Fig. $4.2 \mathrm{c}$ ), we have devised a grid which is mostly a $z$ - level grid, but departs from the $z$ - level constraint near the bottom boundary such that the lowest layer follows the bottom topography. Layers therefore converge near the bottom and allow for good resolution of the bottom boundary layer in most of the domain. The minimum layer thickness is constrained to be $5 \mathrm{~cm}$ at the bottom.

In the first set of experiments the three models were integrated for one year using viscosity and diffusivity values of $A_{M}=10^{4} \mathrm{~m}^{2} \mathrm{~s}^{-1}$ and $A_{H}=0.2 A_{M}$, respec-

tively. This level of horizontal viscosity is that used in the GFDL, Bryan-Cox applications for this resolution (Cox 1975, Bryan and Lewis 1979, Rosati and Myakoda 1988, Derber and Rosati 1989, Bell 1997) and a reduced diffusivity is also typical; we have found that calculations with $A_{H}=A_{M}$ do not differ significantly from those discussed below. However, the reduced diffusivity is generally favorable to the sigma systems to reduce a vertical component of the along - sigma diffusion. Another way to counter along-sigma diffusion is to subtract the (initial in the present case) climatological temperature fields before reckoning along sigma gradients of temperature and salinity and fluxes, and this has been invoked in all three model runs (but is inconsequential for the $z$ - level run). The ultimate solution is to set the along-sigma diffusivity equal to zero which we find possible in many applications of the sigma grid (Mellor et al. 1998, Ezer and Mellor 1999).

Surface elevations, representing the surface velocities, are shown in the left panels and transport streamfunctions, representing the depth integrated velocities, are shown in the right panels in Fig. 4.3 for all three grids. The two sigma models are almost identical. The $z$ - level calculations are also quite similar to the two sigma 

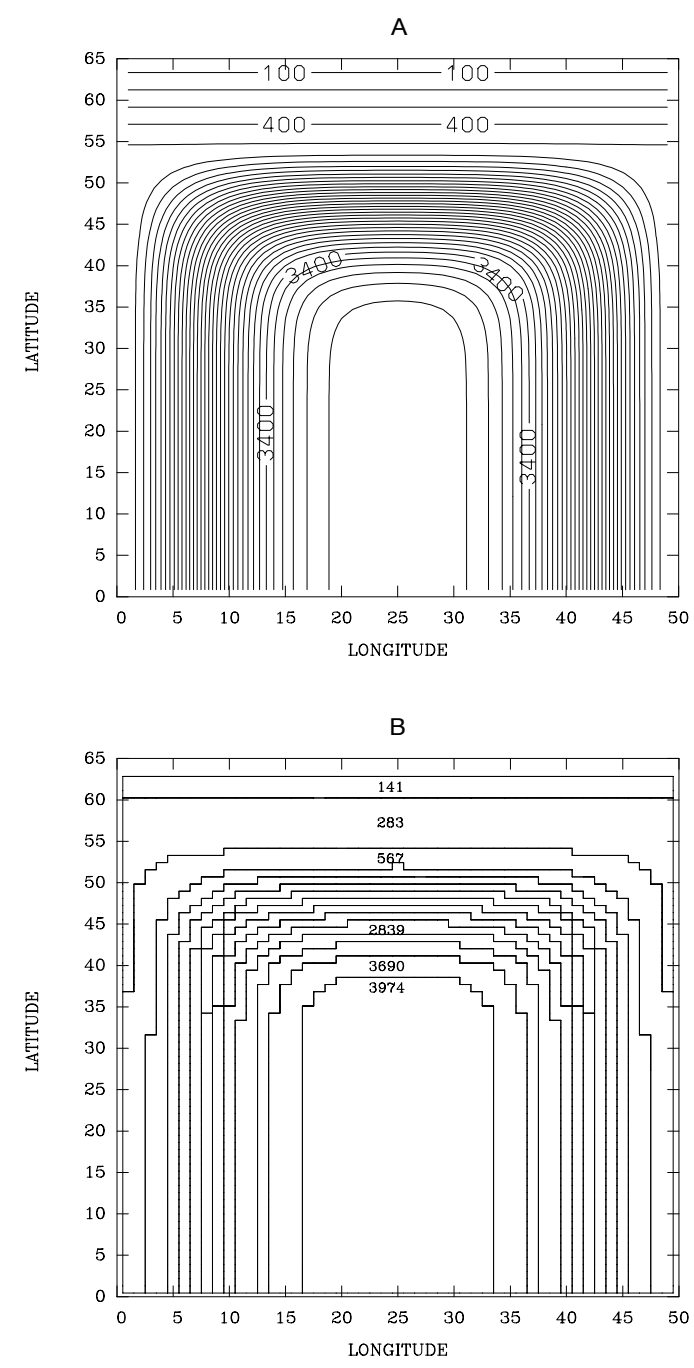

Fig. 4.1. Bottom topography of the model domain, a bowl shaped deep basin with a shallow shelf in the northern part, as represented by different vertical grids: (a) The conventional and the generalized sigma coordinate grids use a continuous bottom depth; the contour interval used in the plot is $100 \mathrm{~m}$. (b) The z-level grid has a step-like topography so that the lines show the location of vertical walls and the numbers show the depth of the bottom of the steps.

models. However, the $z$ - level surface velocities in the shallow northern region are much stronger. In Fig. 4.4 , the temperature cross sections of the three experiments appear to be virtually identical in most of the domain, producing similar upper ocean mixed layers, except near the continental shelf, where only the two sigma 


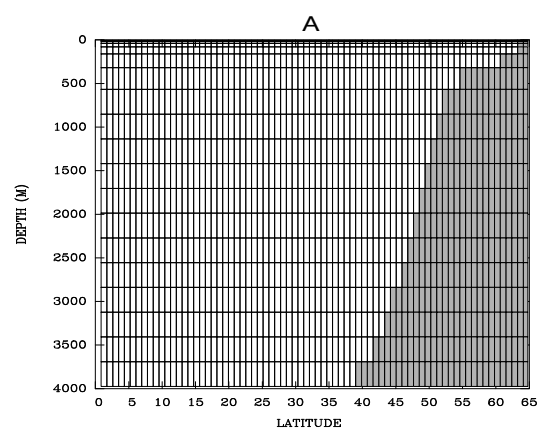

B

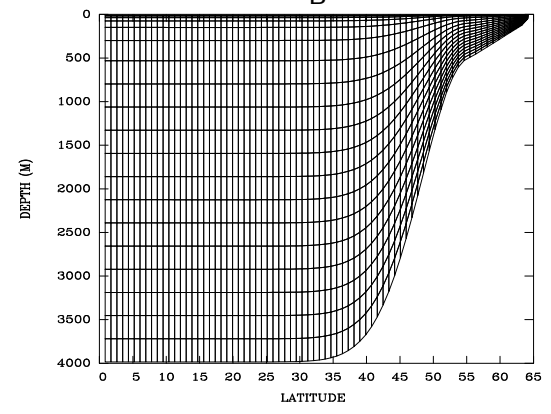

C

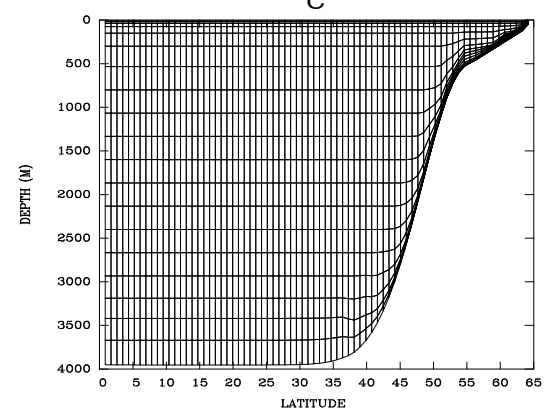

Fig. 4.2. Vertical grids in a nort-south cross section at the center of the domain: (a) the $z$ level grid, (b) the conventional sigma coordinate grid and (c) the generalized sigma grid. The grid in (c) is dominated by z coordinates in the deep ocean, but follows the topography like sigma coordinates near the sloping bottom with layers converging to create a well resolved bottom boundary layer.

models appear to produce also bottom boundary layers. In Fig. 4.5 , and velocity cross sections of the three experiments are fairly similar in the deep ocean, but significantly differ from each other near the continental slope and shelf where the $z$ level grid produces noisy velocity fields.

If the horizontal viscosity and diffusivity are reduced by an order of magnitude, $A_{M}=10^{3} \mathrm{~m}^{2} \mathrm{~s}^{-1}$ and $A_{H}=0.2 A_{M}$, the situation changes. The sigma models tolerate 

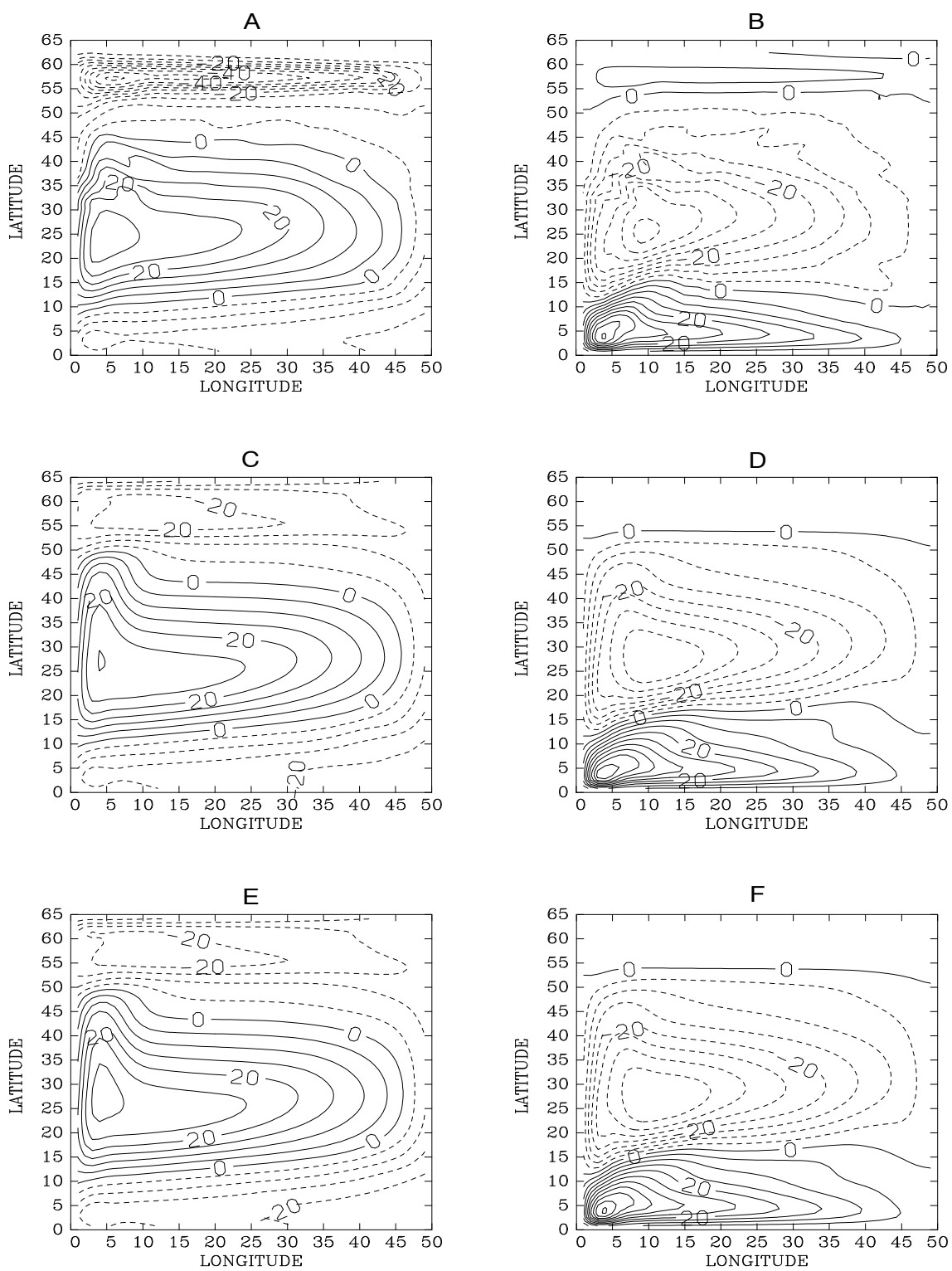

Fig. 4.3. The surface elevation (left panels) and the total stream function (right panels) after 360 days of calculation using a constant viscosity of $A_{M}=10^{4} \mathrm{~m}^{2} \mathrm{~s}^{-1}$; results are shown for the $\mathrm{z}$ - level grid (top panels a and b), the s grid (middle panels $\mathrm{c}$ and $\mathrm{d}$ ) and the generalized $\mathrm{s}$ grid (bottom panels e and f). Contour intervals are $5 \mathrm{~cm}$ in (a), (c) and (e) and $5 \mathrm{~Sv}$ (1 $\left.\mathrm{Sv}=10^{6} \mathrm{~m}^{3} \mathrm{~s}^{-1}\right)$ in (b), (d) and (f).

the reduced values; the $z$ - level model does not as seen in Figs. 4.6, 4.7 and 4.8. At this reduced viscosity and diffusivity, the sigma models exhibit Rossby waves 

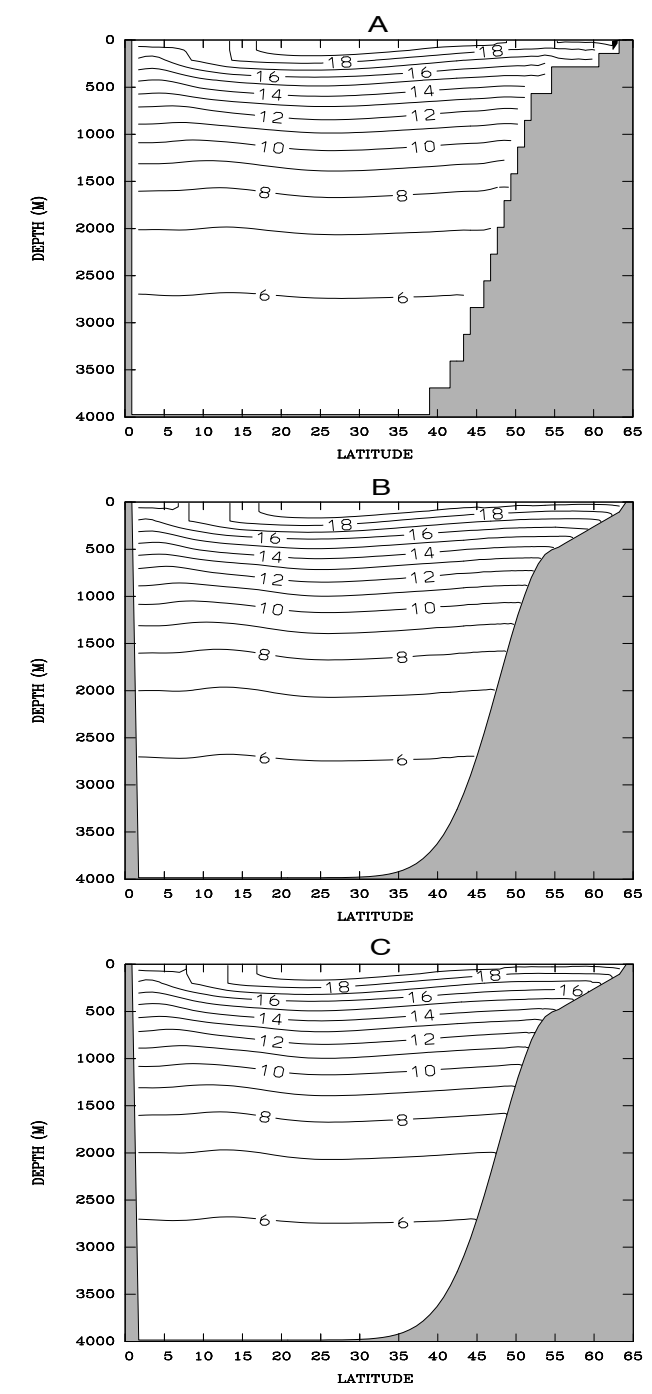

Fig. 4.4. North $\mid$ south temperature cross sections at the center of the domain after 360 days for (a) the $\mathrm{z}$ - grid, (b) the $\mathrm{s}$ grid and (c) the generalized s grid; $A_{M}=10^{4} \mathrm{~m}^{2} \mathrm{~s}^{-1}$.

near the equator (Figs. $4.6 \mathrm{~d}$ and $4.6 \mathrm{f}$ ) that are absent from the high viscosity calculations (Fig. 4.3 ); additional plots (not shown) reveal that the waves propagate westward at a speed of about $20 \mathrm{~km} \mathrm{~d}^{-1}$, about $30 \%$ higher than the speed of the simplest barotropic Rossby wave theory. Experiments (not shown) with even smaller viscosity of $A_{M}=10^{2} \mathrm{~m}^{2} \mathrm{~s}^{-1}$, still show similar results for the sigma grid calculations, but with somewhat more intense Rossby waves.

Other calculations have been performed. They include a lower resolution, $2^{\circ} \times 2^{\circ}$ , on a $26 \times 38 \times 11$ grid and the same topography, initial conditions and surface 

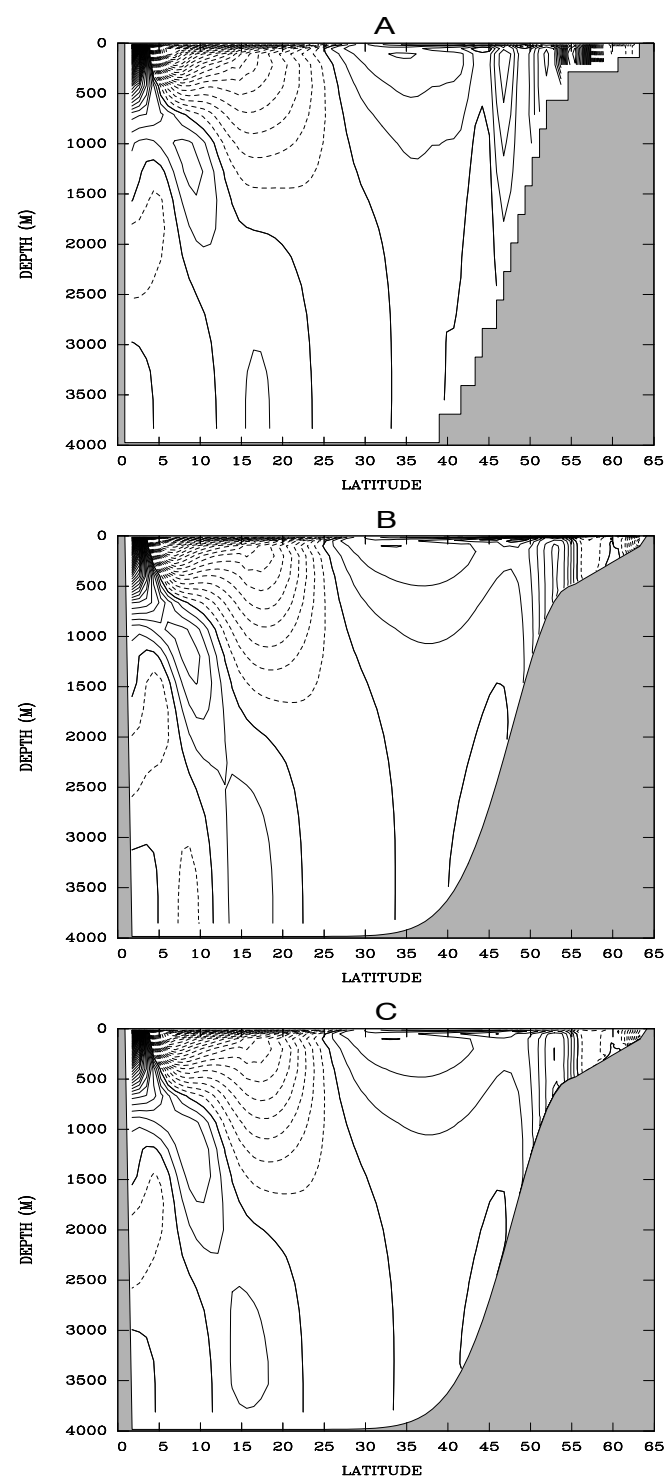

Fig. 4.5. North-south zonal velocity cross sections at the center of the domain after 360 days for (a) the $\mathrm{z}$ - grid, (b) the $\mathrm{s}$ grid and (c) the generalized $\mathrm{s}$ grid; $\mathrm{A}_{\mathrm{M}}=10^{4} \mathrm{~m}^{2} \mathrm{~s}^{-1}$. Contour interval is $0.5 \mathrm{~cm} \mathrm{~s}^{-1}$; dashed contours represent negative values and westward flows.

forcing as before. The viscosity was raised to $A_{M}=5 \times 10^{5} \mathrm{~m}^{2} \mathrm{~s}^{-1}$ and $A_{H}=0.2 A_{M}$. The results were quite similar to the higher resolution, high viscosity results. However, the $z$ - level grid produced numerically divergent results which was repaired by increasing the number of vertical grid points from 11 to 21 . 

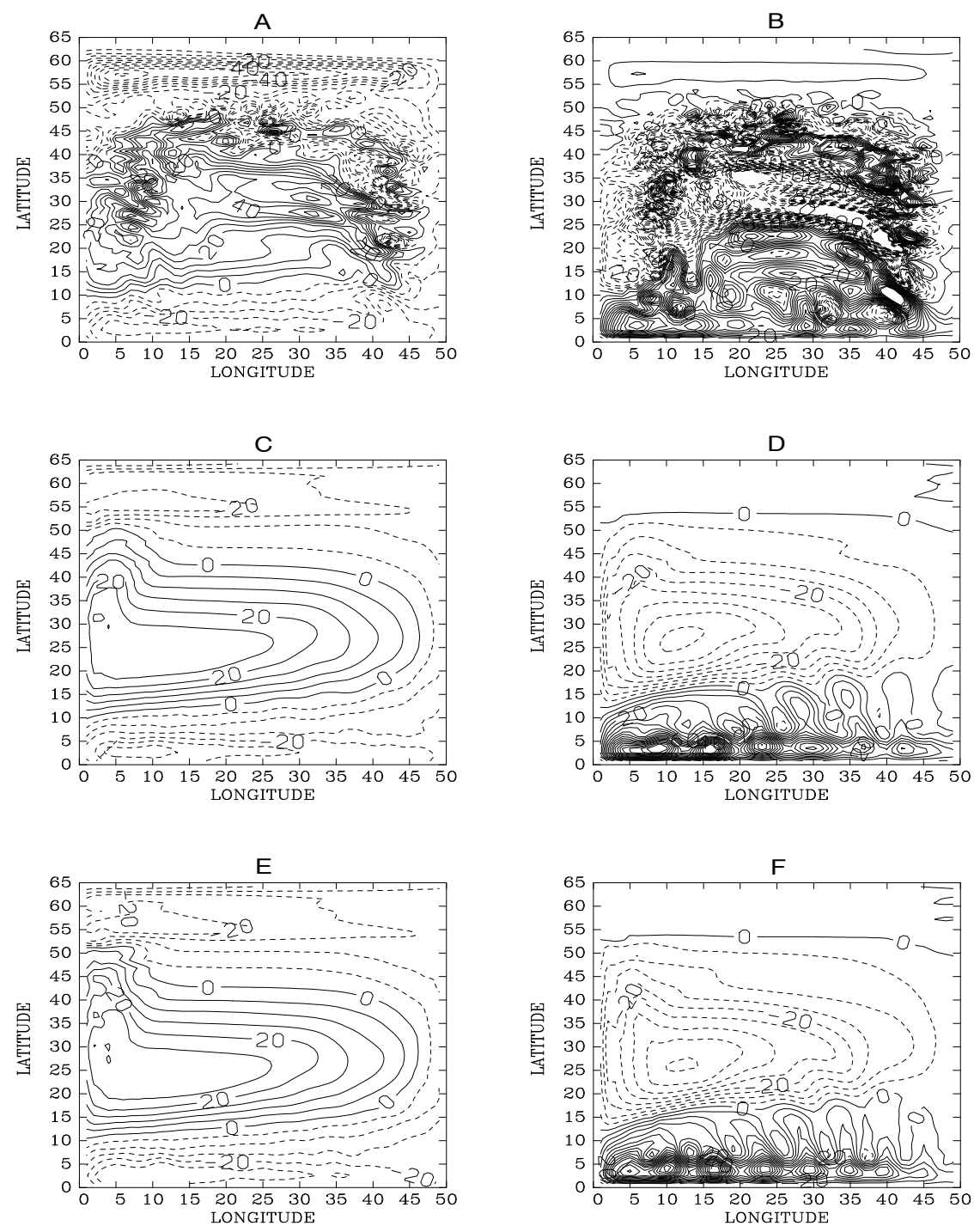

Fig. 4.6. Same as Fig. 4.3 , but for the low viscosity case with $A_{M}=10^{3} \mathrm{~m}^{2} \mathrm{~s}^{-1}$.

This is the place to also mention that POM usually operates with a non-linear, Smagorinsky viscosity and diffusivity which has the advantage that its empirical constant is non-dimensional and nearly resolution independent and yields low values in regions where velocities are low. As noted by Derber and Rosati (1989), the GFDL model cannot tolerate the Smagorinsky formulation and we have also found this to be the case in the present $z$ - level model. 


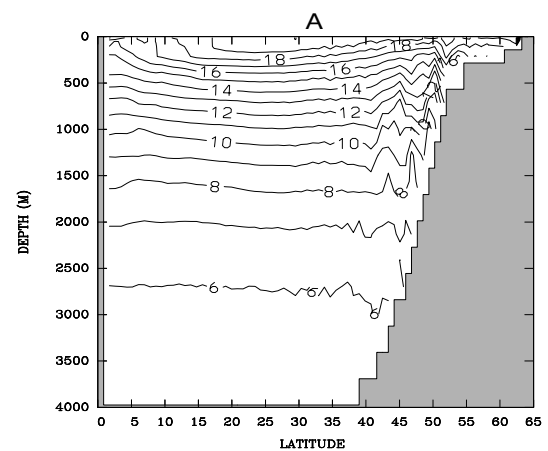

B
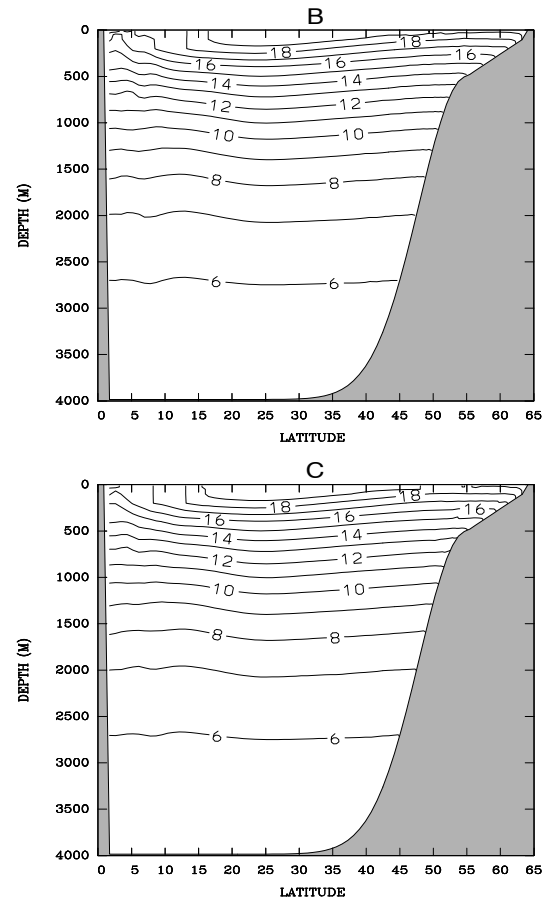

Fig. 4.7. Same as Fig. 4.4 , but for the low viscosity case with $A_{M}=10^{3} \mathrm{~m}^{2} \mathrm{~s}^{-1}$.

\subsection{Errors}

The source of sigma coordinate pressure gradient error is easily identified (Haney, 1991) although the error itself has not been fully understood until recently (Mellor et al. 1994, 1998). It is known that the error can be greatly reduced by subtracting the area averaged density before evaluating density gradients and performing the baroclinic integrals in (14) and (15) or (22) and (23). By running the 

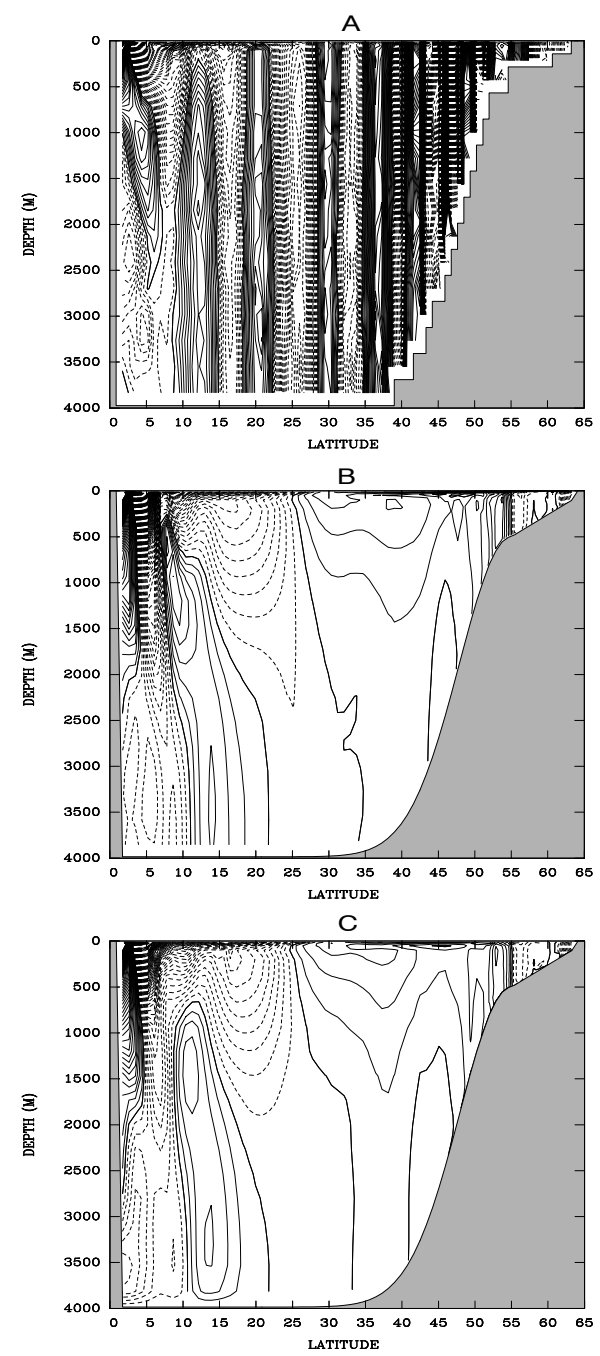

Fig. 4.8. Same as Fig. 4.5 , but for the low viscosity case with $A_{M}=10^{3} \mathrm{~m}^{2} \mathrm{~s}^{-1}$.

straight $\sigma$ model with no wind forcing and with no subtraction of the area averaged density, the maximum velocity and rms errors were approximately $2.5 \mathrm{~cm} \mathrm{~s}^{-1}$ and $0.15 \mathrm{~cm} \mathrm{~s}^{-1}$ in the high viscosity case and $5.0 \mathrm{~cm} \mathrm{~s}^{-1}$ and $0.30 \mathrm{~cm} \mathrm{~s}^{-1}$ in the low viscosity case; the maximum errors were located in the shallowest regions along the eastern and western boundaries. The maximum velocity errors in the generalized sigma model were about the same although the prior expectation was that they would be smaller. In both cases, the maximum vertically averaged velocities were 
similar to the rms velocity errors. The corresponding errors in the $z$ - level case were, of course, nil.

After subtraction of the area averaged density, the errors in the sigma models were also nil. Of course, after the model has run with wind forcing and baroclinic density structure has formed, there must exist some quite small but hard-to-evaluate sigma errors.

However, it is apparent from our results that, with wind forcing, errors in the $z$ level model, may be much larger than in the $\sigma$ grid, in particular, in shallow regions and if viscosity is not high enough. Applying a computational stability analysis to one - dimensional, advection-diffusion equation,

$$
\phi_{i+1}-\phi_{i-1}=2 R^{-1}\left(\phi_{i+1}-2 \phi_{i}+\phi_{i-1}\right)
$$

(Bryan et al. 1975) derived the stability condition $R<2$, where $R=u \Delta x / A$ is the grid cell Reynolds number. Apparently, the step structure of the $z$ - level grid excites this mode ( $R$ varies spatially but is of the order 2 for the high viscosity runs, but is much larger for the low viscosity runs) whereas the $\sigma$ grids do not. Bell (1997) has produced a more complex analysis which identifies vorticity errors due to step structure.

\subsection{Other Grids}

We have looked at and compared only two conventional grids and a third somewhat unconventional, generalized sigma, or s - coordinate grid. (It should be mentioned however, that in the particular application tested here, the superiority of the $\mathrm{s}$ - coordinate system over the standard $\sigma$ - coordinate system has not been demonstrated). However, the possible grid structures are infinite. For example, there is no reason not to have a sigma-like grid in one portion of a model domain and a $z$ level in another portion where, for example, the topography in a low resolution model might be especially steep. Given the model structure described in section 4.5 , it would be possible to create an isopycnal model by creating a single enabling subroutine. The attendant problems of surface and bottom layer representation seem daunting to us, however. Other possibilities are an adaptive vertical coordinate system to resolve the surface mixed layer or an underlying sharp spring and summertime thermocline such as observed in the Great Lakes (Bedford and Schwab 1998).

\subsection{Summary}

There are two main thrusts to this paper. The first thrust is the creation of a model algorithm whose vertical coordinate system is quite general; this algorithm invites future inventiveness with other grids beyond the preliminary tests presented in this 
paper. The second thrust is a model intercomparison study of conventional $z$ - level and $\sigma$ - coordinate vertical grids and one unconventional generalized sigma grid. The intercomparisons are unique in that all aspects of the models are identical except for the vertical grid specification. One finding is that the sigma grids can tolerate much smaller levels of horizontal viscosity and diffusivity. In shallow water, errors arise in the $z$ - level grid which, depending on viscosity (which itself may be considered a source of error), are of a different nature and obviously much larger than errors due to the sigma pressure gradient error.

In the final analysis, the straight $\sigma$ coordinate grid may be a good choice for many ocean applications, including basin-scale climate calculations (Ezer and Mellor 1997). Song and Haidvogel (1994) claim that adequate resolution of the surface layers are a significant advantage of the s - coordinate grid. However, if the vertical grid spacing is, say, logarithmically reduced near the surface to adequately resolve the surface layer in the deepest portions of the model domain, then the only penalty of the $\sigma$ grid is that, in the shallower portions, the surface layers are over resolved. In any case, the new algorithm, developed here for a widely used sigma coordinate ocean model (POM), provides a tool to further investigate the differences between different grids, and provides more flexibility for users to optimize their grids for particular applications.

\section{Acknowledgments}

This research was supported by the Office of Naval Research grant N0014-930037 and by the computational support and facilities of NOAA's Geophysical Fluid Dynamics Laboratory. 


\section{References}

Bedford, K. and D. J. Schwab, 1998. Recent developments in the Great Lakes Forecasting System (GLFS) - performance evaluation. Proceeding: Second Conference on Coastal Atmospheric and Oceanic Prediction and Processes, American Meteorological Society, Boston, MA, 45-50.

Bell, M. J. 1997. Vortex stretching and bottom torque in the Bryan-Cox ocean circulation model. Applications T. N. 17, The Met. Office, U.K.

Blumberg, A.F., Mellor, G.L., 1987. A description of a three-dimensional coastal ocean circulation model. In Heaps, N. S. (Ed.), Three-Dimensional Coastal ocean Models, Coastal Estuarine Studies 4, American Geophysical Union, Washington, DC, 1-16.

Bryan, K., 1969. A numerical model for the study of the circulation of the world oceans. J. Comput. Phys., 4, 347-376.

Bryan, K. and L. J. Lewis, 1979. A water mass model of the world ocean. $J$. Geophys. Res., 84, 2503-2517.

Bryan, K., S. Manabe and R. Pacanowski, 1975. A global ocean-atmosphere climate model. Part II. The oceanic circulation. J. Phys. Oceanogr., 5, 3046.

Cox, M. D., 1975. A baroclinic numerical model of the world ocean: preliminary results. Num. Mod. Ocean Circ., National Academy of Science, Washington, DC.

Cox, M. D., 1984. A primitive equation, 3-dimensional model of the ocean. GFDL Ocean Group Tech. Rep. No. 1, GFDL, Princeton, NJ, 141 pp.

Derber, J. and A. Rosati, 1989. A global ocean data assimilation. . J. Phys. Oceanogr., 19, 1333-1347.

DYNAMO Group, 1997. DYNAMO: Dynamics of North Atlantic Models: Simulation and assimilation with high resolution models. Tech. Rep. Nr. 294, Inst. Fur Meereskunde, Univ. Kiel, Kiel, 339 pp.

Ezer, T., 1999. Decadal variabilities of the upper layers of the subtropical North Atlantic: An ocean model study. J. Phys. Oceanogr., 29, 3111-3124.

Ezer, T. and G. L. Mellor, 1994. Continuous assimilation of Geosat altimeter data into a three-dimensional primitive equation Gulf Stream model. $J$. Phys. Oceanogr., 24, 832-847.

Ezer, T. and G. L. Mellor, 1997. Simulations of the Atlantic Ocean with a free surface sigma coordinate ocean model. J. Geophys. Res., 102, 15,64715,657 .

Ezer, T. and G. L. Mellor, 2000. Sensitivity studies with the North Atlantic sigma coordinate Princeton Ocean Model. Dyn. Atmos. Oceans, 32, 155208. 
Galperin, B., L. H. Kantha, S. Hasid and A. Rosati, 1988. A quasi-equilibrium turbulent energy model for geophysical flows. J. Atmos. Sci., 45, 55-62.

Gerdes, R., 1993. A primitive equation ocean circulation model using a general vertical coordinate transformation. 1. Description and testing of the model. Journal of Geophysical Research 98, 14,683-14,701.

Haney, R.L., 1991. On the pressure gradient force over steep topography in sigma coordinate ocean models. Journal of Physical Oceanography 21, 610619.

Mellor, G.L., 1996. User's guide for a three-dimensional, primitive equation, numerical ocean model. Program in Atmospheric and Oceanic Sciences, Princeton University, Princeton, N. J., 40 pp.

Mellor, G.L., Blumberg, A. F., 1985. Modeling vertical and horizontal diffusivities with the sigma coordinate system. Monthly Weather Review $113,1380-1383$.

Mellor, G. L., Yamada, T., 1982. Development of a turbulent closure model for geophysical fluid problems. Review of Geophysics 20, 851-875.

Mellor, G.L., Ezer, T., Oey, L.-Y., 1994. The pressure gradient conundrum of sigma coordinate ocean models. Journal of Atmospheric and Ocean Technology 11, Part 2, 1126-1134.

Mellor, G.L., Oey, L.-Y., Ezer, T., 1998. Sigma coordinate pressure gradient errors and the seamount problem. Journal of Atmospheric and Ocean Technology 15, 1122-1131.

Rosati, A. and K. Miyakoda, 1988. A general circulation model for upper ocean simulation. J. Phys. Oceanogr., 18, 1601-1626.

Song, Y. and D. Haidvogel, 1994. A semi-implicit ocean circulation model using a generalized topography-following coordinate system. J. Comput. Phys., 115, 228-244.

Spall, M. A. and A. R. Robinson, 1990. Regional primitive equation studies of the Gulf Stream meander and ring formation region. J. Phys. Oceanogr., 20, 985-1016.

Willems, R. C., S. M. Glenn, M. F. Crowley, P. Malanotte-Rizzoli, R. E. Young, T. Ezer, G. L. Mellor, H. G. Arango, A. R. Robinson and C.-C. A. Lai, 1994. Experiment evaluates ocean models and data assimilation in the Gulf Stream. EOS, Trans. American Geophysical Union, 75, 385-394.

Winton, M., R. Hallberg and A. Gnanadesikan, 1998. Simulation of densitydriven frictional downslope flow in z-coordinate ocean model. J. Phys. Oceanogr., 28, 2163-2174. 